\title{
El mundo digital. A la búsqueda de una nueva perspectiva personal alrededor del nuevo entorno de Internet del futuro
}

\author{
F.A.M. van der Reep \\ Inholland University of Applied Science, Rotterdam, Países Bajos y \\ Senior strategist en KPN Consulting
}

\section{Internet: ¿te mueve o es tu movimiento? ${ }^{1}$}

¿Qué le parece el título de este artículo? Probablemente, todos reaccionamos según nuestros valores. Nuestro marco de referencia y nuestro conjunto de cogniciones determina cómo vemos el mundo. Sin duda, usamos nuestro cerebro para ver, no nuestros ojos. Y somos lo que vemos.

En mi opinión, Internet añade otra dimensión a este enfoque. Internet contiene una cantidad de información tan apabullante que no tenemos más remedio que ser más conscientes de nosotros mismos y de nuestros propios valores: no hay vida ni crecimiento sin raíces. De lo contrario nos veremos arrastrados por Internet y seremos dirigidos en lugar de dirigir nuestra propia vida.

Voy a esbozar qué sucede en ese mundo digital desde diferentes puntos de vista para que cada cual se forje su propia opinión al respecto. Suelo utilizar referencias holandesas. Pero no porque Holanda sea especial o única. Estoy convencido de que en otras regiones se aplican las mismas tendencias y es fácil para usted, querido lector, encontrar referencias en su propia región.

Creo que en el futuro, la consecuencia de la digitalización de los flujos de información tendrá un impacto mucho más molesto de lo que ahora estamos

\footnotetext{
${ }^{1}$ Frans van der Reep es estratega senior en Getronics Consulting y profesor de Digital World en la Universidad de Ciencias Aplicadas de Holanda (INHolland). Este artículo se puede leer por sí mismo, pero también en conjunto con Reep, F van der, «Social media and social companies», BPtrends.com, noviembre de 2010. El impacto organizativo de Internet y la forma en que Internet cambia la organización del trabajo se ha estudiado más a fondo en Van der Reep, F (2005), «From Schedule push to Reality pull», European Retail Digest, Oxford University, número 48, Invierno 2005, páginas 33-37, reimpreso en IT Management Select, volumen 15, Invierno 2009-2010, páginas 36-41.
} 
percibiendo. Basta con mirar el mundo de los negocios, la forma en que los mercados y los consumidores se compartimentan. La forma de hacer política, cómo damos forma a las organizaciones. Cómo el periodismo civil añade contenido al periódico. La manera en la que son capturados los delincuentes y, por ejemplo, cómo la gente logra encontrarse, juntarse, unirse. Y cómo, en la sociedad, tratamos de encontrar un nuevo equilibrio entre la hipocondría social y nuevas formas de liderazgo.

Parece que se está desarrollando un Gobierno 2.0 en el que el poder político puede estar organizado de una manera diferente y en el que se utiliza un enfoque más colaborativo. Los poderes más obvios de los gobiernos (al menos en Europa) se abandonaron hace tiempo. La cadena se ha invertido, y a los equipos autogestionados y todo tipo de formas de autoorganización se les llama «referéndum» (Shirky, 2008:123)².

Pienso que mientras el viejo mundo se acabó no encontramos un nuevo orden totalmente establecido. Eso crea un vacío de poder, que podemos usar para bien o para mal. Como resultado, éste también es un momento de decisiones y de asumir responsabilidades personales: ¿cuáles son mis valores?, ¿qué me motiva? y ¿cómo quiero expresar estos valores?.

Una mirada de conjunto nos muestra que al igual que los últimos avances tecnológicos han alcanzado al gran público, la digitalización, la Web 2.0 y la web semántica y sus discursos tienen un impacto que afecta a muchas disciplinas científicas y a muchas facetas de la vida: la economía, la psicología, la sociología y la antropología, por nombrar sólo algunas. Estos desarrollos han impuesto un nuevo entorno donde las viejas reglas han caído: y afecta a las universidades, a los secretarios de Estado del país, o a los departamentos de una empresa, donde el último avance es llamado generalmente Gestión de procesos de negocio (Business Process Management-BPM).

La reciente innovación tecnológica que tuvo un impacto similar, creo, fue la introducción de la píldora en los años 60 . Aquel avance también estuvo acompañado de estructuras sociales totalmente nuevas y cambió nuestra forma de pensar. Y también fue un proceso a largo plazo acompañado de muchos debates, que aún hoy siguen abiertos después de 50 años.

Internet aumenta nuestras capacidades, tanto las creativas como las reaccionarias. En ambos papeles, la web asume la función de un organizador de la inteligencia colectiva; basta pensar en Google y cómo están digitalizando los libros. Internet estimula el reaccionario administrador del trabajo y la vida que llevamos dentro. Al administrador del trabajo y la vida le gusta documentar toda la información, no quiere ni perder ni perderse nada, reflexiona constantemente y conduce, por ejemplo, a cantidad de documentación sensible de nuestro comportamiento en público. Internet ofrece la posibilidad de reaccionar, que se materializa simplemente porque hay mucho ante lo que reaccionar. En esta

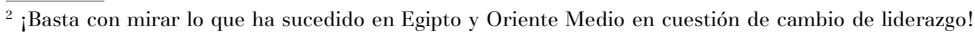


modalidad reactiva de comportamiento se vive desde fuera hacia adentro y en esta modalidad reactiva las acciones como la optimización, la adaptación y la copia son necesarias. Para el mundo de los negocios, éste es el mundo del control y de las mejores prácticas. Un mundo de gestión científica en el que tratamos de adaptarnos al futuro sobre la base de experiencias pasadas y con la ayuda de la ciencia, teniendo en cuenta todos los riesgos que se pueden pensar ahora mismo. Éste es también el mundo del análisis de escenarios.

Por otro lado, Internet estimula la creatividad y nos hace diseñadores de vida. Será más fácil encontrar a otras personas para poder, juntos, lograr nuestros sueños. Éste es el mundo del networking y del trabajo conjunto desde dentro hacia fuera. Esto hace referencia al creador que llevamos dentro. Ése es el mundo del emprendedor que trabaja por su cuenta, una comunidad de rápido crecimiento en Holanda de 800.000 emprendedores en la actualidad que se constituye en parte sustancial del mercado de trabajo holandés.

\section{Internet impulsa la introspección sobre las actitudes y valores de nuestra vida}

La información de Internet no sólo nos facilita la reflexión utilizando la web como un archivo de toda una vida. Internet también nos permite explorar nuevas posibilidades, nuevas oportunidades y hacer nuevas combinaciones. Al crearse el mundo digital, la formación de nuevas redes que como individuo activen iniciativas desde un nuevo punto de vista, inspirado en la cercanía y vinculado a ella, es más fácil y más barato. Esto requiere gente que abandone la comodidad de su propio entorno y se atreva a leer desde el interior hacia el exterior en una curva de aprendizaje. En este caso, Internet sirve como una herramienta que apoya la formación de nuestra propia vida, donde asumimos la responsabilidad de nuestras propias decisiones.

En la actualidad, probablemente necesitaremos ambas cosas, como seres humanos y también desde la óptica de los negocios y hasta desde la perspectiva nacional. Como siempre, es el arte de encontrar el equilibrio y encontrar el justo medio. Sin embargo, creo que el futuro está en el punto de vista creativo, ya que es lo que caracteriza a los seres humanos como creadores que se expresan.

Nuestra forma reactiva de pensar está fuertemente determinada por los hábitos, los patrones, los supuestos, los marcos personales, el miedo al rechazo y por la búsqueda o el temor a las reacciones positivas y negativas de nuestro entorno. Sin embargo, atreverse a crear significa no tener en cuenta certezas externas formalizadas, dejar de lado el pensamiento establecido, y atreverse a mantener una postura propia, entre otras cosas, siendo consciente de nuestros propios talentos, formas de pensar y modelos. Internet está haciendo nuestro mundo tan flexible y dinámico, tan abierto y perpetuamente beta, que las certezas externas son cada vez más extrañas. De hecho, mañana será diferente. 
Apartarnos del pensamiento establecido también nos permite redefinir los negocios como parte de un todo mayor, como parte de un ecosistema. Permite una orientación más amplia, más integrada o, si se desea utilizar esta palabra, más holística. Para cubrir esta perspectiva sobre la vida y el trabajo, Peter Senge et al. introdujeron el término «presencia» (Senge et al., 2006). Con este término, los autores describen la elección de una participación consciente en un territorio cambiante, en el que las personas sin prejuicios están abiertas a las ideas de los demás.

¿Su objetivo es evitar los errores del pasado? Ese es el punto de vista reactivo o reaccionario por el que muchas empresas optan en la actualidad: prestar atención a las mejores prácticas, a la eficiencia, al uso de software para la planificación de recursos empresariales (ERP), a los estudios de mercado y la inteligencia empresarial que incluye el CRM y el VRM (gestión de relaciones con proveedores), a la Ley Sarbanes Oxley $(\mathrm{SOX})^{3}$, a la orientación al control y comparaciones de mejores prácticas entre empresas. Lo que intento señalar es que este es sólo uno de los más de un centenar de puntos de vista posibles. Utilizando este punto de vista de fuera hacia dentro, la empresa toma un camino que debe seguirse fuera de la empresa, haciéndose realmente reactiva.

Cuando la empresa toma sus acciones reaccionando al entorno, al final el alma de la empresa se pierde y la empresa no será más que la ejecutora de las tareas inventadas por otros, adoptando una postura desconfiada y reactiva en el mundo. Se deja llevar por el mercado y no crea nuevos mercados por sí misma. Ya no existe un diseño real. No es nada intrínsecamente malo y hasta reporta algo de dinero en la actualidad. Una empresa así utilizará Internet como un bibliotecario usaría su biblioteca. Impulsados por la voluntad de sobrevivir vemos un enfoque en la búsqueda y combinación inteligente, pero con una fuerte tendencia a sistemas mentales cerrados y bien ordenados, a la reflexión y a la gestión (control) del riesgo. ¿Les suena?

Pero ¿qué pasa si lo exterior es cada vez menos fijo y ofrece menos garantías? ¿Qué pasa si China llega y nos obliga a reducir nuestros gastos en un $70 \%$ en cinco años? Sin duda, todo lo que se transmite por cable, con el aumento constante del ancho de banda, debe poder competir con China, Singapur, Filipinas y Vietnam.

¿A qué nos podemos agarrar en el rápido y flexible mundo digital donde cada día es diferente? ¿Se atreve usted a confiar en sí mismo? ¿Cuál es la base de una estrategia del océano azul, una nueva dirección que sale de la nada? Otro punto de vista es que Internet le invita como comerciante o emprendedor a valerse por sí mismo. Junto a la perspectiva de fuera hacia dentro, aparece una nueva perspectiva orientado desde dentro hacia fuera. Una perspectiva que, para mí, activa nuestras competencias más poderosas. Esto es tan válido para usted como para mí, para empleados y departamentos, y también para empre-

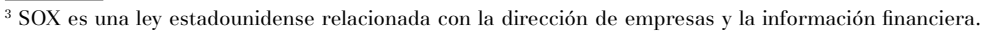


sas y estados. Al pensar de dentro hacia fuera se activan habilidades creativas y empresariales dentro de la organización. Entonces, ya no nos interesan las mejores prácticas y el comportamiento de me too (de seguidor). Optamos por las futuras prácticas y determinamos nuestras propias decisiones y actividades. Damos forma a nuestra propia vida. Escrutamos y pensamos en sistemas abiertos y probablemente utilizamos software libre, redes sociales y wikis para hacer un seguimiento de los demás dentro de la organización. Desde este punto de vista, podemos dar, sin tener la sensación de que estamos malgastando o perdiendo algo. Vemos la cooperación como una competencia fundamental. ¿Quién es nuestro amigo? ¿Con quién compartimos, nos aliamos y nos enlazamos?

Un gran crecimiento requiere unas raíces fuertes. ¿Cuáles son nuestras raíces, o, dicho de otra manera, nuestros valores? ¿Y las redes y la digitalización de nuestro mundo influyen en esa orientación de los valores? ¿Van a cambiar o simplemente Internet nos hace más conscientes de ello porque cada vez es más explícita para nosotros? ¿La función de emprendedor ha cambiado debido a Internet? ¿Qué hace Internet con nuestros valores y cómo situamos Internet respecto a nuestros valores? Este es un ámbito académico antropológico (digital) completamente nuevo y fascinante.

Como metáfora, podríamos considerar Internet como un gran centro de medios de comunicación: ¿dirigimos el cotarro o nos dejamos llevar?

Centrándonos en el valor individual, propio, comenzará a haber más diversidad en la sociedad. ¿A dónde nos llevará eso? No lo sé. ¿Más confianza en la sociedad, o tal vez menos? ¿La sociedad se volverá más tribal? ¡Desde luego es fascinante!

\section{Internet y la organización del trabajo}

Al mirar la figura 1, podemos ver dos latidos del corazón, reaccionar y crear, en los cuadrantes enfrentamiento (counter) y encuentro (en-counter). En el cuadrante de enfrentamiento, la gente eligió los límites, adaptándose a los marcos existentes y a la gestión, mando y control como un mecanismo de coordinación. En el contexto de los negocios, básicamente sobreviremos por medio del camuflaje: dentro de la organización, no somos más que el título de nuestro cargo. No es relevante quiénes somos. En este cuadrante, la invitación permanente es adaptarse a la empresa: es lo que hay. Eso es reactivo. Este es el mundo de los certificados: Así es como lo hacemos aquí. Jaap Peters y Judith Pouw hablan de «cría intensiva de humanos» (Peters y Pouw, 2004:122-127). En este sentido, Internet se está utilizando como una herramienta eficaz para la distribución de la información. Las soluciones dentro del cuadrante enfrentamiento se caracterizan a menudo por la división del trabajo y, como tal, por la compartimentación, que se traduce en un entorno en el que nadie es realmente capaz de asumir la responsabilidad, porque no hay tal responsabilidad o quizá sólo la hay en tér- 
minos abstractos. Todo el mundo está optimizando su propia parcela y no hay nadie que supervise la totalidad o que siquiera se preocupe por hacerlo. Por lo tanto, la cooperación se convierte en un reto y, por desgracia, a menudo se traduce en la búsqueda de intereses propios paralelos y en un sálvese quien pueda. Este es el mundo de los procesos mentales de la victoria y de la competencia y de la supervivencia del más preparado: comer o ser comido.

Contrarrestar es la forma en que la mayoría, aproximadamente el $65 \%$ en Holanda, de las empresas y las instituciones gubernamentales se están organizando. La excelencia en el control y la excelencia operativa, centrarse en la estabilidad y la eficiencia (TQM, Lean Thinking y Six Sigma) tienden a reducir los costes; de hecho haciendo buenos los errores del pasado.

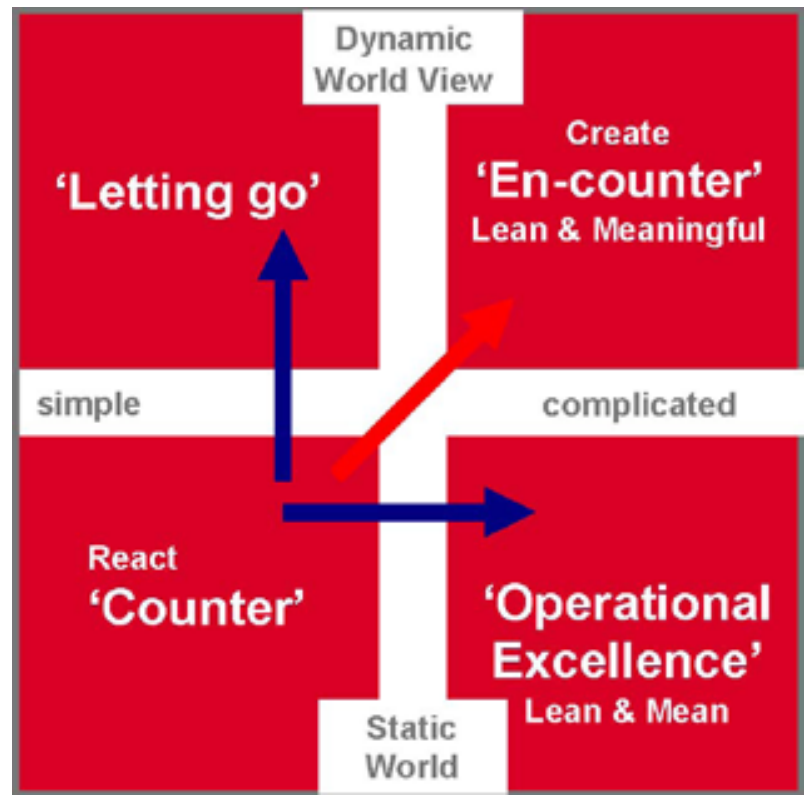

El mundo desde fuera hacia dentro es básicamente reactivo. Es básicamente un mundo de camuflaje, ya que es la estrategia de supervivencia individual lo que es tan característico de nuestro modo reactivo de conciencia. Este es el mundo del control, de los cargos y la conducta basada en reglas, el mundo de las prácticas correctas, implícita pero básicamente destinadas a evitar la responsabilidad personal.

Podemos prosperar en el mundo de encuentros, en el mundo desde dentro hacia fuera. Encontrar significa que las empresas y/o los individuos se organizan en comunidades cambiantes, como organizaciones en red eficientes para abastecer una demanda de mercado que cambia rápidamente y creando sus propios mer- 
cados. Aquí está por ejemplo, la llamada estrategia del océano azul. La estrategia de supervivencia del individuo aquí ya no es el camuflaje y la adaptación. Ésta es una estrategia que juega con nuestros puntos fuertes. Esta estrategia requiere un compromiso personal y una salida del armario del camuflaje. El modo creativo requiere que adoptemos una posición en la vida, que abandonemos el puerto seguro y que nos embarquemos en el viaje de nuestra vida empresarial. Por supuesto, las tormentas puede ser parte de este viaje... Pero esto es así.

Muchos emprendedores que trabajan por su cuenta trabajan así. La estrategia de supervivencia aquí ya no es el camuflaje, sino ponerse de pie, una nueva forma de trabajo y procesos dirigidos por humanos, que muestran quiénes somos, lo que se traduce en la supervivencia de los más cooperativos.

El motor básico de la cooperación siempre es compartir y dar. No deberíamos olvidar eso $^{4}$.

También podemos ilustrar este esquema a través de la figura 2 (véase a continuación), en la que la distinción indicada entre personalidades abierta y cerrada, así como asertiva y no asertiva coincide con la subdivisión de las personalidades de Jung (abierta frente a cerrada y un carácter asertivo frente a uno no asertivo), lo que refleja su visión de que todo lo vivo es energía y por lo tanto refleja una polaridad, que es tan característica de nuestro tiempo.

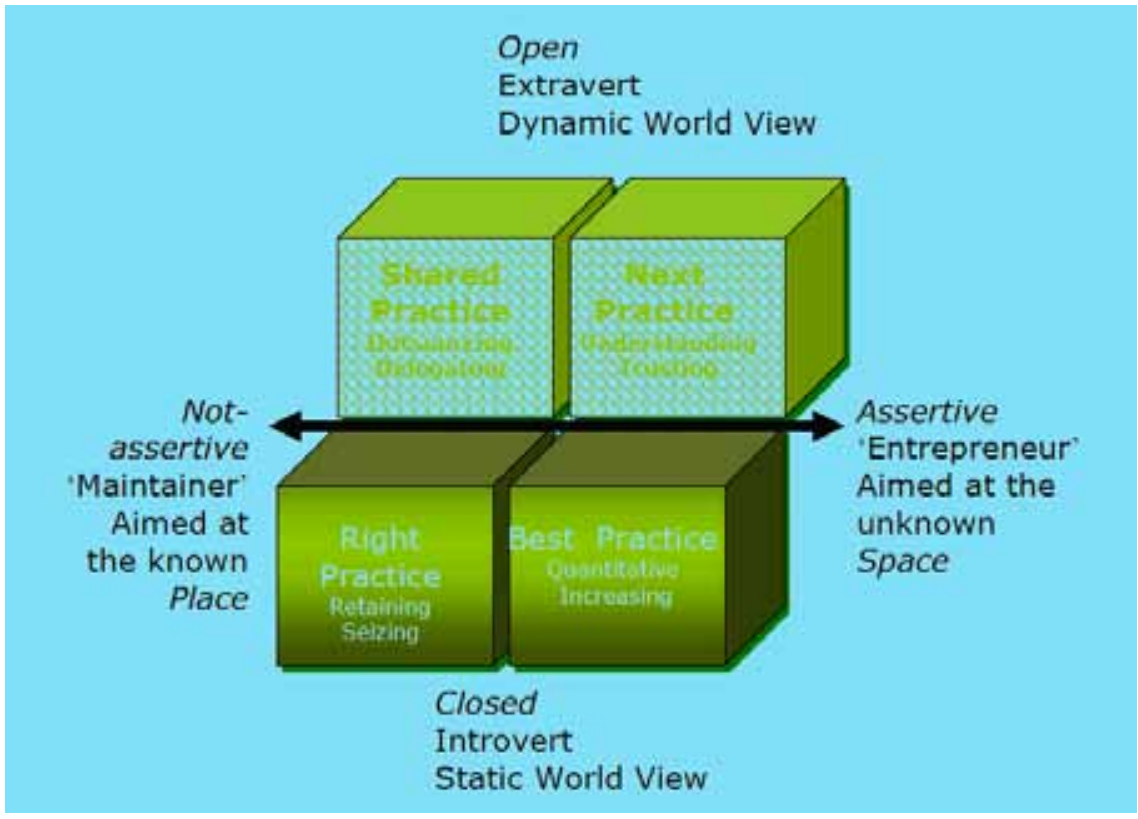

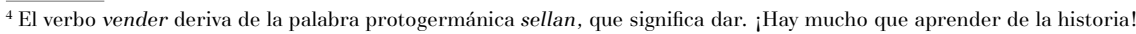


La palabra lugar (aimed at the known place) a la izquierda de la figura 2 se refiere al pensamiento encerrado en la esquina inferior izquierda: Pensar dentro de marcos, dentro de la caja. La palabra espacio (aimed at the unknown space) a la derecha de la figura se refiere al pensamiento ilimitado en la esquina superior derecha, fuera de la caja ${ }^{5}$.

Las perspectivas enfrentar y encontrar de la figura 1 son análogas de alguna forma, les siguen de la misma manera. Las formas flexibles de cooperación en la formación de las comunidades beneficiarias sustituyen a la jerarquía y al control central como mecanismo de coordinación dentro de las empresas.

Espero que la presión de los plazos esté cada vez más reemplazada por el arrastre de la demanda real ${ }^{6}$. Las grandes empresas están cada vez más rodeadas de otras más pequeñas que tienen, proporcionalmente hablando, un poder considerable. Cuestiones éticas aparte existen buenas razones para esto. Internet reduce el coste de crear cooperación en al menos un 50\% en comparación con las estructuras jerárquicas como medio para facilitar la cooperación.

En lo que respecta a la orientación del valor subyacente, parece que las competencias individuales y la manera honesta e integral en la que alguien percibe estas oportunidades y las comunica son gradualmente más importantes para el éxito en un mundo con tanta movilidad. La autenticidad, la credibilidad y la coherencia en las acciones personales, así como el enfoque en las fortalezas propias marcarán la pauta. Anselm Grün afirma que el conocimiento de uno mismo, el conocimiento de la naturaleza, la creatividad y la fantasía humanas son requisitos previos para el liderazgo, que comienza con liderarse a uno mismo (Grün, 2004: 52-55). Como tal, el desarrollo empresarial, el desarrollo personal y/o dirigir el país es una cuestión de puntos de vista y de cómo se pueden percibir, por ejemplo, los negocios, las redes sociales, la ciencia y la política desde muchas perspectivas diferentes pero iguales, reactiva (cuando es el gerente), o creativa (cuando nos referimos al emprendedor). Les invito a adoptar y entender un punto de vista diferente aunque sea sólo una vez. Para entenderse a sí mismo, a sus colegas dentro de la empresa, a sus amigos, a sus hijos o a cualquier otra persona. Creo que nuestra capacidad de comprensión es exactamente lo que marca la diferencia entre la evolución hacia el capitalismo compasivo con respeto mutuo y una economía de pirañas en la que todos compiten entre sí. Ver más posturas de la historia, por ejemplo, la postura de nuestro competidor, permite la comunicación verdadera y genera el respeto mutuo.

Internet realmente nos permite dar el paso personal introduciendo «la época personal» (Mieke y Lovink, eds., 2010) y nos muestra quiénes somos y ¡nos convierte realmente en diseñadores de vida! ¡Emocionante!

\footnotetext{
${ }^{5}$ Por supuesto, uno podría argumentar que la expresión fuera de la caja sigue siendo un término dentro de la caja ya que aún existe la caja. No tengo una solución a este problema.

${ }^{6}$ En Holanda existen actualmente pruebas con trenes que circulan con tanta frecuencia entre Amsterdam y Eindhoven, ya $\sin$ horarios fijos, que el tren casi se convierte en un transporte público del cual tira la demanda como con el taxi.
} 


\section{Referencias}

Applegate, Lynda M. et al. (2004). Corporate Information Strategy and Management. New York: McGraw Hill.

Gerritsen, Mieke y Geert Lovink (2010). Everyone is a designer in the age of social media. Ámsterdam: BIS Publishers.

Grün, Anselm (2004). Bezielend Leidinggeven. Kampen: Ten Have/Lannoo.

In 't Veld, Roel (2010). Knowledge Democracy: Consequence for Science, Politics and Media. Berlín/Heidelberg: Springer.

Peters, Jaap y Pouw, Judith (2004). Intensieve menshouderij: hoe kwaliteit oplost in rationaliteit. Culemborg: Central Boekhius.

Reep, Frans van der (2005). Vuistregels voor succesvol innovaren. En: Overheid Innovatief, número 48, p. 32-33.

Reep, Frans van der (2005). About an Analogous Life in a Digital World. Rotterdam: IINholland.

Senge, Peter et al, (2006). Presence, an Exploration of profound Change in People. New York: Doubleday.

Shirky, Clay (2008). Here Comes Everybody, Revolution doesn't happen when Society adopts new Technologies, it happens when Society adopts New Behaviors. Nueva York: The Penguin Press.

\section{Referencia de este artículo}

Van der Reep, F.A.M. (2011). El mundo digital. A la búsqueda de una nueva perspectiva personal alrededor del nuevo entorno de Internet del futuro. En: adComunica. Revista Científica de Estrategias, Tendencias e Innovación en Comunicación, $\mathrm{n}^{0}$ 2. Castellón: Asociación para el Desarrollo de la Comunicación adComunica, Universidad Complutense de Madrid y Universitat Jaume I, 205213. 